Pacific Journal of Mathematics

A CHARACTERIZATION OF THE GAUSSIAN DISTRIBUTION

Ja 


\title{
A CHARACTERIZATION OF THE GAUSSIAN DISTRIBUTION IN A HILBERT SPACE
}

\author{
JefFrey L. SpIELMAN
}

In this paper we consider the case in which random variables $X_{j}$ take values in a real, separable Hilbert space $\mathscr{H}$. We look at a linear form $\Sigma A_{j} X_{j}$ where each $A_{j}$ is a bounded linear operator in $\mathscr{H}$. We then assume that this linear form is identically distributed with a monomial and form conditions under which it is possible to deduce that the common distribution of the random variables is the Gaussian distribution.

The study of identically distributed linear forms of independent and identically distributed random variables has been undertaken by several authors. J. Marcinkiewicz studied linear forms in which all moments of the random variables are assumed to exist. He then proved that the common distribution of the random variables was the Normal distribution. R. G. Laha and E. Lukacs have considered the case where one of the linear forms is a monomial. They have obtained characterizations of the Normal distribution for both the case when the variance is assumed finite and when no assumption is made concerning the variance.

1. Statement of the main result. Suppose now that $X_{1}, X_{2}, \cdots$ is a sequence (possibly finite) of independent, identically distributed, nondegenerate $\mathscr{H}$-valued random variables, where $X_{1}$ has a finite variance (i.e. $\operatorname{Var} X_{1}<+\infty$ ). Let $A_{1}, A_{2}, \cdots$ be a sequence of $1-1$ bounded linear operators in $\mathscr{H}$, with the following two properties:

$$
\sum_{j}\left\|A_{i}\right\|^{2}<+\infty \text { and } \sum_{j} A_{j}^{*} A_{j} \geqq I
$$

and

$$
\sup _{j}\left\|A_{j}\right\|<1
$$

We note that in the above $A_{j}^{*}$ represents the adjoint of $A_{j}$ and that the inequality $\Sigma_{j} A_{j}^{*} A_{j} \geqq I$ is true in the sense of positivedefiniteness. (For example, see page 313 of [7].)

Our goal is to prove the following theorem. 
THEOREM 1. Suppose that $\sum_{I} A_{j} X_{l}$ converges with probability one. If $\sum_{1} A_{1} X_{1}$ has the same distribution as $X_{1}$, then $X_{1}$ has a Gaussian distribution.

In $\$ 2$ we will prove an important preliminary result (Theorem 2). Then in $\$ 3$ we will present the proof of Theorem 1.

2. A preliminary result. In this section we will prove the following result.

THEOREM 2. Let $X_{1}, X_{2}, \cdots$ be a sequence (possibly finite) of independent, identically distributed, nondegenerate, $\mathscr{H}$-valued random variables. Suppose that the sum $\Sigma_{j} A_{j} X_{j}$ exists with probability one, where $A_{1}, A_{2}, \cdots$ are bounded linear operators in $\mathscr{H}$, with $\sup _{j}\left\|A_{l}\right\|<1$.

If $\sum_{,} A_{1} X_{,}$has the same distribution as $X_{1}$, then $X_{1}$ has an infinitely divisible distribution.

Note. The hypotheses of Theorem 2 are somewhat weaker than the hypotheses of Theorem 1 .

Before beginning the proof of Theorem 2, let us fix some notation.

Let $\varphi(y)$ be the common characteristic functional of $X_{1}, X_{2}, \cdots$. Then $\varphi(y)=\mathscr{E} e^{\imath\left\langle X_{1}, y\right\rangle}$ for all $y \in \mathscr{H}$, where $\mathscr{E}$ denotes mathematical expectation.

The characteristic functional of $A_{1} X_{,}$is then given by:

$$
\mathscr{E} e^{i\left\langle A, X_{p}, y\right\rangle}=\mathscr{E} e^{\imath\left\langle X_{,} A^{*}, y\right\rangle}=\varphi\left(A_{j}^{*} y\right)
$$

where $A_{j}^{*}$ denotes the adjoint operator of $A_{j}$.

Now, suppose that $\sum_{j} A_{j} X_{j}$ has the same distribution as $X_{1}$. Then equation (3) gives us:

$$
\varphi(y)=\prod_{1} \varphi\left(A^{*} y\right), \quad \text { for all } \quad y \in \mathscr{H},
$$

where the product converges uniformly on bounded spheres. (See Theorem 4.4, pg. 171 of [5].)

Since $\sum_{j} A_{j} X_{j}$ converges, then $\sum_{j=n}^{\infty} A_{j} X_{j}$ converges, with probability one, to the origin of $\mathscr{H}$ as $n \rightarrow \infty$. (Of course, if $X_{1}, X_{2}, \cdots$ is a finite sequence, the preceding statement is unnecessary.)

Thus, it is possible to choose $N_{0}$ for any $\epsilon>0$, such that $P\left\{\left\|\Sigma_{j=N+1}^{\infty} A, X,\right\|>\epsilon\right\}<\epsilon$, whenever $N \geqq N_{0}$. Let $\varphi_{N}(y)$ denote the characteristic functional of $\sum_{\jmath=N+1}^{\infty} A_{l} X_{l}$. Then using equation (4), we have:

$$
\varphi(y)=\varphi\left(A_{1}^{*} y\right) \cdots \varphi\left(A_{N}^{*} y\right) \varphi_{N}(y) .
$$


Proof of Theorem 2. We assume that $\Sigma_{j} A_{j} X_{j}$ has the same distribution as $X_{1}$. Then equation (5) holds. If we replace $y$ by $A_{,}^{*} y$ in equation (5), we obtain:

$$
\varphi\left(A_{,}^{*} y\right)=\varphi\left(A_{1}^{*} A_{j}^{*} y\right) \cdots \varphi\left(A_{N}^{*} A_{j}^{*} y\right) \varphi_{N}\left(A_{j}^{*} y\right)
$$

for each $j=1,2, \cdots, N$.

Combining equations (5) and (6) we have:

$$
\varphi(y)=\prod_{j=1}^{N} \varphi\left(\left(A_{l}^{*}\right)^{2} y\right) \cdot \prod_{j \neq k} \varphi\left(A_{j}^{*} A_{k}^{*} y\right) \prod_{j=1}^{N} \varphi_{N}\left(A_{j}^{*} y\right) \varphi_{N}(y)
$$

If we repeat the above process $n$ times, we get the following result:

$$
\varphi(y)=\Pi \varphi\left(A_{j_{1}}^{*} \cdots A_{j_{n}}^{*} y\right) \prod_{k=1}^{n-1} \Pi \varphi_{N}\left(A_{j_{1}}^{*} \cdots A_{J_{n-k}}^{*}\right) \varphi_{N}(y) .
$$

The product on the right hand side of equation (7) consists of $N^{n}+N^{n-1}+\cdots+N+1$ factors, where each of the subscripts $j_{1}, \cdots, j_{n}$ can take any of the values $1, \cdots, N$ with repetitions allowed.

Thus, equation (7) says that $X_{1}$ is distributed as the sum of $k_{n}=\sum_{k=0}^{n} N^{k}$ independent, $\mathscr{H}$-valued random variables, $Y_{n, k} \quad(k=$ $\left.1,2, \cdots, k_{n}\right)$, for any positive integer $n$.

We will now show that $Y_{n, k}$ is a uniformly infinitessimal collection of random variables. That is, we will show that for any $\epsilon>0$, $\sup _{1 \leqq k \leqq k_{n}} P\left\{\left\|Y_{n k}\right\|>\epsilon\right\} \rightarrow 0$ as $n \rightarrow \infty$. Once this has been established, the infinite divisibility of $X_{1}$ will follow from Corollary 6.2, page 199 of [5].

Consider the factors on the right hand side of equation (7). Let $\epsilon>0$ be given. By definition $\varphi_{N}(y)$ is the characteristic functional of $\sum_{j=N+1}^{\infty} A, X_{j}$ and $P\left\{\left\|\sum_{j=N+1}^{\infty} A_{j} X_{j}\right\|>\epsilon\right\}<\epsilon$, for all $N \geqq N_{0}$.

Consider now a factor of the form $\varphi_{N}\left(A_{{ }_{1}}^{*} A_{j_{2}}^{*} \cdots A_{j_{n-k}}^{*} y\right)$. This is the characteristic functional of

$$
A_{j_{n-k}} \cdots A_{l_{2}} A_{j_{1}} \sum_{j=N+1}^{\infty} A_{j} X_{j}
$$

Also,

$$
\begin{aligned}
P\left\{\| A_{j_{n-k}} \cdots\right. & \left.A_{j_{2}} A_{j_{1}} \sum_{j=N+1}^{\infty} A_{j} X_{j} \|>\epsilon\right\} \\
& \leqq P\left\{\left\|A_{j_{n-k}}\right\| \cdots\left\|A_{j_{2}}\right\| \cdot\left\|A_{j_{1}}\right\| \cdot\left\|\sum_{j=N+1}^{\infty} A_{j} X_{j}\right\|>\epsilon\right\} \\
& \leqq P\left\{\left\|\sum_{j=N+1}^{\infty} A_{j} X_{j}\right\|>\epsilon\right\}<\epsilon \text { whenever } \quad N \geqq N_{0},
\end{aligned}
$$

since $\sup _{j}\left\|A_{j}\right\|<1$. 
Finally, we consider a factor of the form $\varphi\left(A_{j_{1}}^{*} \cdots A_{j_{n}}^{*} y\right)$, which is the characteristic functional of $A_{j_{n}} \cdots A_{j_{1}} X_{1}$. Set $\alpha=\sup _{\jmath}\left\|A_{j}\right\|$.

Then

$$
\begin{aligned}
P\left\{\left\|A_{j_{n}} \cdots A_{j_{1}} X_{1}\right\|>\epsilon\right\} & \\
& \leqq P\left\{\left\|A_{j_{n}}\right\| \cdots\left\|A_{j_{1}}\right\| \cdot\left\|X_{1}\right\|>\epsilon\right\} \\
& \leqq P\left\{\left\|X_{1}\right\|>\frac{\epsilon}{\alpha^{n}}\right\} .
\end{aligned}
$$

Now choose an integer $N^{\prime}$ such that $P\left\{\left\|X_{1}\right\|>\epsilon / \alpha^{n}\right\}<\epsilon$, whenever $n \geqq N^{\prime}$. (This is possible because $0<\alpha<1$ ). Set $n_{0}=\max \left\{N_{0}, N^{\prime}\right\}$.

Hence, we have shown that $P\left\{\left\|Y_{n k}\right\|>\epsilon\right\}<\epsilon$, for all $k=1,2, \cdots, k_{n}$, whenever $n \geqq n_{0}$. Therefore, the collection $Y_{n k}$ is uniformly infinitesimal and $X_{1}$ is infinitely divisible. This completes the proof of the theorem.

3. Proof of the main result. For convenience, we now will make the assumption that $X_{1}, X_{2}, \cdots$ are symmetric random variables. Since the common distribution of these random variables is infinitely divisible, the common characteristic functional, $\varphi(y)$, has a unique Levy-Khintchine representation given by:

$$
\ln \varphi(y)=-\frac{1}{2}\langle S y, y\rangle+\int(\cos \langle x, y\rangle-1) d L(x)
$$

where $S$ is an $S$-operator (a nonnegative, self-adjoint compact operator on $\mathscr{H}$, with a finite trace), and $L$ is a $\sigma$-finite measure with finite mass outside every neighborhood of the origin and with the property that

$$
\int_{\|x\| \leqq 1}\|x\|^{2} d L(x)<+\infty .
$$

(see [5], page 181.)

Furthermore, since $X_{1}, X_{2}, \cdots$ have finite variance, $\varphi(y)$ has a unique Kolmogorov representation, given by:

$$
\ln \varphi(y)=-\frac{1}{2}\langle S y, y\rangle+\int_{\mathscr{E}\{\{0\}} \frac{\cos \langle x, y\rangle-1}{\|x\|^{2}} d K(x)
$$

where $S$ is an $S$-operator and $K$ is a finite measure on $\mathscr{H}$. (See [6].) By equations (4) and (8) we have:

$$
\begin{aligned}
-\frac{1}{2} \sum_{j}\left\langle S A_{i}^{*} y, A_{j}^{*} y\right\rangle+ & \sum_{j} \int\left(\cos \left\langle x, A_{j}^{*} y\right\rangle-1\right) d L(x) \\
& =-\frac{1}{2}\langle S y, y\rangle+\int(\cos \langle x, y\rangle-1) d L(x) .
\end{aligned}
$$


Also,

$$
\begin{aligned}
& -\frac{1}{2} \sum_{J}\left\langle S A_{j}^{*} y, A_{j}^{*} y\right\rangle+\sum_{J} \int\left(\cos \left\langle x, A_{j}^{*} y\right\rangle-1\right) d L(x) \\
& =-\frac{1}{2}\left\langle\sum_{j} A_{j} S A_{j}^{*} y, y\right\rangle+\sum_{j} \int\left(\cos \left\langle A_{j} x, y\right\rangle-1\right) d L(x) \\
& \quad=-\frac{1}{2}\left\langle\sum_{j} A_{l} S A_{,}^{*} y, y\right\rangle+\sum_{j} \int(\cos \langle x, y\rangle-1) d L A_{J}^{-1}(x) .
\end{aligned}
$$

It is not difficult to show that $\Sigma_{l} A_{l} S A_{,}^{*}$ is an $S$-operator. Also, it is clear that $L A_{j}^{-1}$ is the $\sigma$-finite measure which occurs in the Levy-Khintchine representation of $A_{j} X_{j}$, for each $j$.

We denote by $\mathscr{B}$, the class of Borel sets in $\mathscr{H}$. Then the measure $K_{l}$, defined by:

$$
K_{l}(D)=\int_{D}\|x\|^{2} d L A_{i}^{-1}(x), \quad \text { for all } \quad D \in \mathscr{B}
$$

is the finite measure which occurs in the Kolmogorov representation of $A_{j} X_{i}$, for each $j$.

Since $X_{1}, X_{2}, \cdots$ have finite variance,

$$
\int\|x\|^{2} d L(x)<+\infty . \quad(\text { See [6].) }
$$

By equation $(10), \ln \Pi_{j} \varphi\left(A_{j}^{*} y\right)$

$$
=-\frac{1}{2}\left\langle\sum_{j} A_{j} S A_{j}^{*} y, y\right\rangle+\sum_{j} \int_{\{x \neq 0\}} \frac{(\cos \langle x, y\rangle-1)}{\|x\|^{2}} d K_{j}(x) .
$$

We note that

$$
\begin{aligned}
& \sum_{j} \int_{\{x \neq 0\}}\left|\frac{\cos \langle x, y\rangle-1}{\|x\|^{2}}\right| d K_{,}(x) \leqq \sum_{l} \int_{\{x \neq 0\}} \frac{\|x\|^{2}\|y\|^{2}}{\|x\|^{2}} d K_{j}(x) \\
& \quad=\|y\|^{2} \sum_{l} \int_{\{x \neq 0\}} d K_{J}(x)=\|y\|^{2} \sum_{j} \int\|x\|^{2} d L A_{J}^{-1}(x) \\
& \quad=\|y\|^{2} \sum_{l} \int\left\|A_{l} x\right\|^{2} d L(x) \leqq\left(\sum_{J}\left\|A_{J}\right\|^{2}\right)\|y\|^{2} \int\|x\|^{2} d L(x)<\infty
\end{aligned}
$$

because of relations (1) and (11).

Thus we may interchange the integral and summation signs in equation (12) to obtain: 


$$
\ln \prod_{J} \varphi\left(A_{,}^{*} y\right)=-\frac{1}{2}\left\langle\sum_{J} A_{l} S A_{,}^{*} y, y\right\rangle+\int_{\{x \neq 0\}} \frac{\cos \langle x, y\rangle-1}{\|x\|^{2}} d\left(\sum_{,} K_{l}(x)\right) .
$$
have:

Then, by the uniqueness of the Kolmogorov representation, we

$$
\sum_{l} A_{l} S A_{l}^{*}=S \quad \text { and } \quad \sum_{l} K_{l}=K
$$

From the second of these relations, $\Sigma_{J} K_{J}(\mathscr{H})=K(\mathscr{H})$, which leads to the following sequence of equations.

$$
\begin{aligned}
& \sum_{J} \int\|x\|^{2} d L A_{j}^{-1}(x)=\int\|x\|^{2} d L(x) \\
& \sum_{j} \int\left\|A_{j} x\right\|^{2} d L(x)=\int\|x\|^{2} d L(x) \\
& \int\left[\sum_{j}\left\|A_{j} x\right\|^{2}-\|x\|^{2}\right] d L(x)=0 \\
& \int\left[\left\langle\sum_{l} A_{,}^{*} A_{j} x, x\right\rangle-\langle x, x\rangle\right] d L(x)=0 .
\end{aligned}
$$

In view of relation (4), it must then be true that

$$
L\left\{x: \sum_{1}\left\|A_{1} x\right\|^{2}-\|x\|^{2}>0\right\}=0 \text {. }
$$

We note that for $n$ a positive integer, $\sum_{J=1}^{n} A_{l} X_{,}$has characteristic functional $\Pi_{j=1}^{n} \varphi\left(A_{j}^{*} y\right)$, and

$$
\begin{aligned}
\ln \prod_{j=1}^{n} \varphi\left(A_{j}^{*} y\right)= & -\frac{1}{2}\left\langle\sum_{j=1}^{n} A_{j} S A_{j}^{*} y, y\right\rangle \\
& +\int(\cos \langle x, y\rangle-1) d\left(\sum_{j=1}^{n} L A_{l}^{-1}(x)\right) .
\end{aligned}
$$

Thus $\sum_{j=1}^{n} L A_{j}^{-1}$ converges weakly, outside closed neighborhoods of $0 \in \mathscr{H}$, to $L$, as $n \rightarrow \infty$. (See [5], page 189).

It now becomes necessary to state and prove two technical lemmas.

Lemma 1. For any $\epsilon>0$,

$$
\int_{\|x\|>\epsilon}\|x\|^{2} d L(x)=\sum_{j} \int_{\left\|A_{j} x\right\|>\epsilon}\left\|A_{j} x\right\|^{2} d L(x) .
$$


Proof. Let $\epsilon_{1}$ and $\epsilon_{2}$ be positive constants with $\epsilon_{1}<\epsilon_{2}$. Define a function $f(x)$ by:

$$
f(x)= \begin{cases}\|x\|^{2}, & \epsilon_{1}<\|x\| \leqq \epsilon_{2} \\ \left(\epsilon_{2}\right)^{2}, & \|x\|>\epsilon_{2} .\end{cases}
$$

Then $f(x)$ is bounded and continuous. Thus by comment (14),

$$
\int_{\|x\|>\epsilon_{1}} f(x) d L(x)=\lim _{n \rightarrow \infty} \sum_{j=1}^{n} \int_{\|x\|>\epsilon_{1}} f(x) d L A_{j}^{-1}(x)
$$

which implies that

$$
\sum_{j=1}^{n} \int_{\epsilon_{1}<\|x\| \leqq \epsilon_{2}}\|x\|^{2} d L A_{j}^{-1}(x)+\left(\epsilon_{2}\right)^{2} \sum_{j=1}^{n} L A_{j}^{-1}\left\{\|x\|>\epsilon_{2}\right\}
$$

converges to

$$
\int_{\epsilon_{1}<\|x\| \leqslant \epsilon_{2}}\|x\|^{2} d L(x)+\left(\epsilon_{2}\right)^{2} L\left\{\|x\|>\epsilon_{2}\right\}
$$

as $n \rightarrow \infty$.

But, again because of comment (14), $\sum_{j=1}^{n} L A_{j}^{-1}\left\{\|x\|>\epsilon_{2}\right\}$ converges to $L\left\{\|x\|>\epsilon_{2}\right\}$ as $n \rightarrow \infty$.

Therefore, $\sum_{j=1}^{n} \int_{\epsilon_{1}<\|x\| \leqq \epsilon_{2}}\|x\|^{2} d L A_{j}^{-1}(x)$ converges to

$$
\int_{\epsilon_{1}<\|x\| \leqq \epsilon_{2}}\|x\|^{2} d L(x)
$$

whenever we choose $0<\epsilon_{1}<\epsilon_{2}$.

Let $\epsilon>0$ be given. Let $\epsilon_{n}$ be a strictly increasing sequence of positive numbers, $\epsilon_{n} \uparrow+\infty$, with $\epsilon_{1}>\epsilon$. For convenience we set $\epsilon=\epsilon_{0}$.

Then

$$
\begin{aligned}
\int_{\|x\|>\epsilon_{0}}\|x\|^{2} d L(x) & =\sum_{k=0}^{\infty} \int_{\epsilon_{k}<\|x\| \leq \epsilon_{k+1}}\|x\|^{2} d L(x) \\
& =\sum_{k=0}^{\infty} \sum_{j} \int_{\epsilon_{k}<\|x\|<\epsilon_{k+1}}\|x\|^{2} d L A_{j}^{-1}(x) \\
& =\sum_{j} \sum_{k=0}^{\infty} \int_{\epsilon_{k}<\|x\| \leq \epsilon_{k+1}}\|x\|^{2} d L A_{j}^{-1}(x) \\
& =\sum_{j} \int_{\|x\|>\epsilon}\|x\|^{2} d L A_{j}^{-1}(x)=\sum_{j} \int_{\left\|A_{j} x\right\|>\epsilon}\left\|A_{j} x\right\|^{2} d L(x) .
\end{aligned}
$$

This completes the proof.

Lemma 2. $L\left(\left\{x:\left\|A_{k} x\right\|^{2} \geqq\|x\|^{2}\right.\right.$, for some $\left.\left.k=1,2, \cdots\right\}\right)=L(\{0\})$. 
Proof. Let $k$ be a fixed positive integer.

Set $E_{k}=\left\{x:\left\|A_{k} x\right\|^{2}=\|x\|^{2}\right\}$. Then, using equation (13), $L\left(E_{k}\right)=$ $L\left(E_{k} \cap\left\{x: \Sigma_{l}\left\|A_{l} x\right\|^{2}=\|x\|^{2}\right\}\right)$. is $1-1$.

Thus, $L\left(E_{k}\right)=L\left\{x: \Sigma_{\jmath \neq k}\left\|A_{j} x\right\|^{2}=0\right\}=L(\{0\})$, since each operator $A_{j}$

$$
\text { Similarly, } L\left(\bigcup_{k} E_{k}\right)=L(\{0\}) \text {. }
$$

Using the same type of argument, it is easy to show that for all $k=1,2, \cdots$

$$
L\left\{x:\left\|A_{k} x\right\|^{2}>\|x\|^{2}\right\}=0 .
$$

Combining equations (15) and (16) we are done.

From relations (1) and (13), we see that $L\left\{x:\|x\|^{2} \neq \Sigma_{,}\left\|A_{f} x\right\|^{2}\right\}=$ 0 . Hence, referring to Lemma 1 , it is true that, for all $\epsilon>0$,

$$
\sum_{j} \int_{\|x\|>\epsilon}\left\|A_{j} x\right\|^{2} d L(x)=\sum_{j} \int_{\left.\left\|A_{j}\right\| \|\right\rangle \epsilon}\left\|A_{j} x\right\|^{2} d L(x)
$$

and this implies that

(17) $\sum_{j}\left[\int_{\|x\|>\epsilon}\left\|A_{j} x\right\|^{2} d L(x)-\int_{\left\|A_{x} x\right\|>\epsilon}\left\|A_{j} x\right\|^{2} d L(x)\right]=0$, for all $\epsilon>0$.

But

$$
L\left\{x: x \neq 0 \text { and }\left\|A_{j} x\right\| \geqq\|x\|\right\}=0, \text { for all } j .
$$

Thus, each term in the sum of equation (17) must be nonnegative, which yields:

$$
\int_{\|x\|>\epsilon}\left\|A_{j} x\right\|^{2} d L(x)=\int_{\left\|A_{,} x\right\|>\epsilon}\left\|A_{j} x\right\|^{2} d L(x), \text { for all } \epsilon>0 \text { and all } j .
$$

Or, using equation (18),

$$
\int_{\{\|x\|>\epsilon\} \cap F_{,}}\left\|A_{j} x\right\|^{2} d L(x)=\int_{\left\{\left\|A_{,} x\right\|>\epsilon\right\} \cap F_{j}}\left\|A_{j} x\right\|^{2} d L(x),
$$

for all $\epsilon>0$ and all $j$, where $F_{j}=\left\{x:\left\|A_{j} x\right\|<\|x\|\right\}$, for each $j=1,2, \cdots$.

The above implies that 


$$
\int_{\{\|x\|>\epsilon\} \cap\{\|A, x\| \leq \epsilon\} \cap F_{j}}\|A, x\|^{2} d L(x)=0, \text { for all } \epsilon>0 \text { and all } j,
$$

or,

$$
\int_{\{\|x\|>\epsilon \operatorname{and}\|A, x\| \subseteq \epsilon\}}\left\|A_{j} x\right\|^{2} d L(x)=0, \quad \text { for all } \epsilon>0 \text { and all } j .
$$

So, we must have that

$$
L\left\{x:\|x\|>\epsilon \quad \text { and } \quad\left\|A_{j} x\right\| \leqq \epsilon\right\}=0, \quad \text { for all } \epsilon>0 \text { and all } j .
$$

Consider the set $Q^{+}$of positive rational numbers. Let $k$ be a fixed positive integer.

$L\left[\bigcup_{r \in Q^{+}}\left\{x:\|x\|>r \quad\right.\right.$ and $\left.\left.\quad\left\|A_{k} x\right\| \leqq r\right\}\right]=L\left[\left\{x:\left\|A_{k} x\right\|<\|x\|\right\}=L[\mathscr{H} \backslash\{0\}]\right.$.

Therefore, $L[\mathscr{H} \backslash\{0\}] \leqq \sum_{r \in Q^{+}} L\left\{x:\|x\|>r\right.$ and $\left.\left\|A_{k} x\right\| \leqq r\right\}=0$, by equation (19).

This last relation says that $L$ is degenerate at $0 \in \mathscr{H}$, which means that the common characteristic functional of $X_{1}, X_{2}, \cdots$ is given by:

$$
\ln \varphi(y)=-\frac{1}{2}\langle S y, y\rangle \quad \text { (see Eq. (8)) }
$$

Hence $X_{1}, X_{2}, \cdots$ have a common Gaussian distribution.

Recall that we have assumed $X_{1}, X_{2}, \cdots$ to be symmetric, but it is now easy to extend our result to the general case by using Cramer's Theorem (see page 141 of [1]).

The proof of Theorem 1 is now completed.

\section{REFERENCES}

1. U. Grenander, Probabilities on Algebraic Structures, Wiley, New York (1963).

2. R. G. Laha, On a property of infinitely divisible distributions in a Hilbert space, Acta Math. Acad. Sci. Hungar. 20 (1969), 143-147.

3. R. G. Laha and E. Lukacs, On a linear form whose distribution is identical with that of a monomial, Pacific J. Math., 15 (1965), 207-214.

4. J. Marcinkiewicz, Sur une propriété de la loi de Gauss, Math. Zeitschr., 44 (1949), 612-618.

5. K. R. Parthasarathy, Probability Measures on Metric Spaces, Academic Press, New York (1967).

6. B. L. S. Prakasa Rao, Some characterization theorems for Wiener processes in a Hilbert space, Z. Wahr. verw. Geb., 19 (1971), 103-116.

7. W. Rudin, Functional Analysis, McGraw-Hill, New York (1973).

Received April 2, 1976.

Chicago State University

CHICAGo, IL 60628 



\section{PACIFIC JOURNAL OF MATHEMATICS}

\section{EDITORS}

RichaRd ARENS (Managing Editor)

University of California

Los Angeles, CA 90024

\section{R. A. Beaumont}

University of Washington

Seattle, WA 98105

C. C. Moore

University of California

Berkeley, CA 94720
J. DugunduI

Department of Mathematics

University of Southern California

Los Angeles, CA 90007

R. Finn AND J. Milgram

Stanford University

Stanford, CA 94305

\section{ASSOCIATE EDITORS}
E. F. BECKENBACH
B. H. NeumanN
F. WOLF
K. YoshidA

\section{SUPPORTING INSTITUTIONS}

UNIVERSITY OF BRITISH COLUMBIA

CALIFORNIA INSTITUTE OF TECHNOLOGY

UNIVERSITY OF CALIFORNIA

MONTANA STATE UNIVERSITY

UNIVERSITY OF NEVADA

NEW MEXICO STATE UNIVERSITY

OREGON STATE UNIVERSITY

UNIVERSITY OF OREGON

OSAKA UNIVERSITY

\author{
UNIVERSITY OF SOUTHERN CALIFORNIA \\ STANFORD UNIVERSITY \\ UNIVERSITY OF HAWAII \\ UNIVERSITY OF TOKYO \\ UNIVERSITY OF UTAH \\ WASHINGTON STATE UNIVERSITY \\ UNIVERSITY OF WASHINGTON \\ AMERICAN MATHEMATICAL SOCIETY
}

The Supporting Institutions listed above contribute to the cost of publication of this Journal, but they are not owners or publishers and have no responsibility for its contents or policies.

Mathematical papers intended for publication in the Pacific Journal of Mathematics should be in typed form or offset-reproduced (not dittoed), double spaced with large margins. Underline Greek letters in red, German in green, and script in blue. The first paragraph or two must be capable of being used separately as a synopsis of the entire paper. Items of the bibliography should not be cited there unless absolutely necessary, in which case they must be identified by author and Journal, rather than by item number. Manuscripts, in duplicate, may be sent to any one of the four editors. Please classify according to the scheme of Math. Reviews, Index to Vol. 39. All other communications should be addressed to the managing editor, or Elaine Barth, University of California, Los Angeles, California, 90024.

100 reprints are provided free for each article, only if page charges have been substantially paid. Additional copies may be obtained at cost in multiples of 50 .

The Pacific Journal of Mathematics is issued monthly as of January 1966. Regular subscription rate: $\$ 72.00$ a year (6 Vols., 12 issues). Special rate: $\$ 36.00$ a year to individual members of supporting institutions.

Subscriptions, orders for back numbers, and changes of address should be sent to Pacific Journal of Mathematics, 103 Highland Boulevard, Berkeley, California, 94708.

PUBLISHED BY PACIFIC JOURNAL OF MATHEMATICS, A NON-PROFIT CORPORATION

Printed at Jerusalem Academic Press, POB 2390, Jerusalem, Israel. 


\section{Pacific Journal of Mathematics}

Vol. 68, No. 2

April, 1977

William Allen Adkins, Aldo Andreotti and John Vincent Leahy, An analogue of Oka's theorem for weakly normal complex spaces ........

Ann K. Boyle, M. G. Deshpande and Edmund H. Feller, On nonsingularly

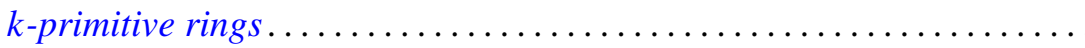

Rolando Basim Chuaqui, Measures invariant under a group of

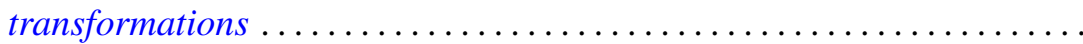

Wendell Dan Curtis and Forrest Miller, Gauge groups and classification of bundles with simple structural group .......................

Garret J. Etgen and Willie Taylor, The essential uniqueness of bounded nonoscillatory solutions of certain even order differential equations

Paul Ezust, On a representation theory for ideal systems

Richard Carl Gilbert, The deficiency index of a third order operator ........

John Norman Ginsburg, $S$-spaces in countably compact spaces using Ostaszewski's method.

Basil Gordon and S. P. Mohanty, On a theorem of Delaunay and some

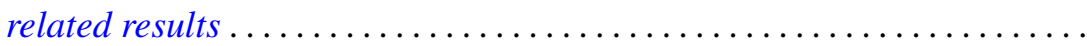

Douglas Lloyd Grant, Topological groups which satisfy an open mapping

theorem.

Charles Lemuel Hagopian, A characterization of solenoids

Kyong Taik Hahn, On completeness of the Bergman metric and its

subordinate metrics. II .

G. Hochschild and David Wheeler Wigner, Abstractly split group extensions.

Gary S. Itzkowitz, Inner invariant subspaces ...............

Jiang Luh and Mohan S. Putcha, A commutativity theorem for

non-associative algebras over a principal ideal domain.

Donald J. Newman and A. R. Reddy, Addendum to: "Rational approximation of $e^{-x}$ on the positive real axis".....

Akio Osada, On the distribution of a-points of a strongly annular function ....

Jeffrey Lynn Spielman, A characterization of the Gaussian distribution in a Hilbert space. .

Robert Moffatt Stephenson Jr., Symmetrizable-closed spaces ...

Peter George Trotter and Takayuki Tamura, Completely semisimple inverse $\Delta$-semigroups admitting principal series . . . . . . . .

Charles Irvin Vinsonhaler and William Jennings Wickless, Torsion free abelian groups quasi-projective over their endomorphism rings...

Frank Arvey Wattenberg, Topologies on the set of closed subsets ... 